\title{
Usos agroindustriales de la hoja de coca en comunidades indígenas del departamento del Cauca, Colombia durante el postconflicto ${ }^{1}$
} Agro-industrial uses of the coca leaf in indigenous communities of Cauca, Colombia in the post-conflict framework Yudith, Caicedo-Domínguez²; Guadalupe, Hoyos-Garcés³; Rocío, Yépez; Diana Karen, Sandoval-Pinedo5;

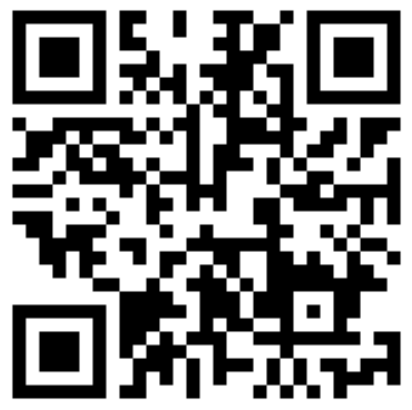

Fecha de recibido: 22-09-2020

Fecha de aceptado: 16-12-2020

\section{(cc) BY-NC-ND}

Esta obra está bajo una licencia de Creative Commons Reconocimiento-NoComercialSinObraDerivada 4.0 Internacional

\section{RESUMEN}

La investigación que se presenta a continuación tuvo el objetivo de plantear las experiencias más significativas relacionadas con los usos agroindustriales lícitos de la hoja de coca, que las comunidades indígenas del departamento del Cauca Colombia han adoptado en el marco del posconflicto. Se dieron a conocer casos exitosos como el generado a raíz de las actividades productivas que se establecieron a través de un proyecto desarrollado por el Servicio Nacional de Aprendizaje (SENA) y la Open Society Foundations, específicamente en la comunidad de Lerma, también el exitoso caso de Coca Nasa. En cuanto a la metodología de la investigación, está se realizó con enfoque cualitativo y exploratorio, como resultado, se encontró que en los últimos cinco años el departamento del Cauca Colombia ha presentado un crecimiento significativo del cultivo de coca, especialmente en los sectores habitados por comunidades indígenas y campesinas quienes han preservado el significado ancestral, alimenticio, medicinal y cultural de la coca. Por último, se concluyó que la agroindustria proveniente de la hoja de coca es viable en este territorio. Sin embargo, las comunidades se enfrentan a múltiples impedimentos para generar esta opción de desarrollo económico y productivo en la región, desde el contexto político y legal.

Palabras claves: Agroindustrial, Cauca Colombia, comunidades indígenas, hoja de coca, postconflicto.

\begin{abstract}
The research presented below had the objective of presenting the most significant experiences related to the licit agroindustrial uses of the coca leaf, which the indigenous communities of the department of Cauca Colombia have adopted in the post-conflict framework. Successful cases such as the one generated as a result of the productive activities that were established through a project developed by the National Learning Service (SENA) and de Open Society Foundations, specifically in the community of Lerma, were also revealed of Coca Nasa. Regarding the research methodology, it was carried out with a qualitative and exploratory approach, as a result, it was found that in the last five years the department of Cauca Colombia has presented a significant growth of coca cultivation, especially in the sectors inhabited by indigenous and peasant communities who have preserved the ancestral, nutritional, medicinal, and cultural meaning of coca. Finally, it was concluded that the agro-industry from the coca leaf is viable in this territory. However, the communities face multiple impediments to generate this option for economic and productive development in the region, from the political and legal context.
\end{abstract}

Keywords: Agroindustry, Cauca Colombia, coca leaf, communities indigenous, post-conflict.

Cómo referenciar este artículo:

Caicedo-Domínguez., Y.; Hoyos-Garcés., G.; Yépez., R.; Sandoval-Pinedo., D., K. (2021). Usos agroindustriales de la hoja de coca en comunidades indígenas del departamento del Cauca, Colombia durante el postconflicto. Revista Politica Globalidad y Ciudadanía, 7(14), 44-62. $\underline{\text { https://doi.org/10.29105/pgc7.14-3 }}$

\footnotetext{
${ }^{1}$ Este artículo es producto del proyecto de investigación "Usos agroindustriales de la hoja de coca como actividad productiva para las comunidades indígenas del departamento del Cauca Colombia en el marco del postconflicto”, financiado por la Universidad Nacional Abierta y a Distancia UNAD. Iniciado en 2017 y finalizado en 2019.

${ }_{2}^{2}$ Universidad Nacional Abierta y a Distancia UNAD, Colombia. Magister en Administración de Organizaciones por la Universidad Nacional Abierta y a Distancia UNAD, Profesora Investigadora de la Universidad Nacional Abierta y a Distancia UNAD. Email: yudithcaicedo6@hotmail.com. Orcid: https://orcid.org/0000-0003-1061-5167

${ }^{3}$ Servicio Nacional de Aprendizaje SENA, Colombia. Especialista en Gerencia de Mercadeo por la Fundación Universitaria de Popayán, líder programa SER SENA regional Cauca Colombia, Email: lupehoyos55@gmail.com, Orcid: https://orcid.org/0000-0002-3255-0643.

${ }^{4}$ Universidad Nacional Abierta y a Distancia UNAD, Colombia. Magister en educación superior por la Universidad Nacional Abierta y a Distancia UNAD Florida, Profesora Investigadora de Universidad Nacional Abierta y a Distancia UNAD. Email: rocio.yepez@unad.edu.co, Orcid: https://orcid.org/00000002-2602-0590.

${ }_{5}^{5}$ Universidad de Guadalajara, México. Estudiante de licenciatura en Derecho en la Universidad de Guadalajara. Email: karens_17@outlook.com, Orcid: https://orcid.org/0000-0001-9601-7453.
} 


\section{1.- INTRODUCCIÓN}

El departamento del Cauca Colombia está situado en el suroeste de Colombia, siendo uno de los departamentos más golpeados por el conflicto armado en el país, por lo que el postconflicto se presenta como un escenario coyuntural con oportunidades para el desarrollo productivo, y consecuentemente económico, a través de unidades de producción que han de permitir a las comunidades, aprovechar las riquezas agrícolas del departamento mediante la agroindustria netamente lícita. Es por lo anterior, y debido a las características climáticas, culturales y de suelos presentes en el Departamento del Cauca Colombia; que esta investigación se enfoca en el cultivo legal de la hoja de coca en este territorio, ya que todos estos factores hacen propicia su siembra, cultivo, crecimiento y posterior comercialización. En ese sentido y con el objetivo de identificar los usos agroindustriales de la coca como materia prima de diversos proyectos productivos alternativos, esta investigación plantea dar continuidad a la realizada por Avendaño, Galvis y Velasco (2017) desde el grupo de investigación Prometeo "Retos y desafíos de las actividades productivas en las comunidades indígenas del departamento del Cauca Colombia en el marco del postconflicto" en la cual, mediante el uso de herramientas prospectivas como el método DELPHI, se construyeron posibles escenarios a los que se enfrentarían las actividades productivas de las comunidades objeto de estudio, formulando también un plan estratégico y un plan de acción en el que se tuvieron en cuenta las estrategias, metas, objetivos, actores y un plan de seguimiento que sirviera a dicha población. Lo anterior, con el fin de hacer frente a los retos y desafíos que traería la firma de la paz y el fin del conflicto armado en Colombia, así como su contribución a las actividades productivas de las comunidades indígenas del departamento del Cauca Colombia. Se identificaron 8 programas estratégicos entre los cuales se encuentra los usos alternativos de la hoja de coca, programa que, a pesar de ser considerado un reto, conlleva a un escenario prometedor en pro del desarrollo y mejoramiento de la calidad de vida de estas comunidades.

\section{FUNDAMENTO TEÓRICO}

De acuerdo con Rojas (2002):

La coca está vinculada al género erythroxilum dentro el orden vegetal, la hoja de coca es cultivada originariamente en Perú, Colombia y Bolivia, aunque sus antecedentes milenarios permiten suponer que en algún momento fue conocida en casi todas las regiones de la América india. Se estima que existen aproximadamente alrededor de 250 variedades de coca. (p.35) 
Así mismo, la coca como la describe Ecu Red (2017):

Es un arbusto de la familia de las Eritroxilàceas, originaria de las escarpadas estrabaciones de los Andes amazonicos, propia de Amèrica del Sur, con nutrientes medicinales y alimenticios. Sus cuatro variedames màs notables son "Lambram", "Mollecoca", "Fusiforme", "ovoide" con hojas alternas a ovadas, enteras de estìpulas axiliares y de flores blanquecinas. Este arbusto se cultiva en las praderas de montañas o en terrazas de altiplanos, en clima tropical y subtropical (párr.3)

La estructura biológica de la hoja de coca está compuesta de 14 principios activos, de uno de esos sale la cocaína. Los otros trece tienen otras posibilidades, tales como usos medicinales, alimenticios, aromáticos o para el trato de la tierra. Sin embargo, la alta rentabilidad en la comercialización de la cocaína ha generado una explotación desmedida de la hoja de coca y condenó de paso a toda la planta a estar en el listado de narcóticos. Este concepto erróneo castró las exploraciones de los componentes benéficos de la hoja de coca, pero existen innumerables investigaciones alrededor del mundo que dan cuenta de sus características benéficas y capacidades agroindustriales, incluso, el fundador de la medicina peruana, Hipólito Unanue ha escrito al respecto. De alguna manera, la prohibición y la persecución de la cocaína ha entorpecido el desarrollo de proyectos productivos que logren extraer todo el potencial de esta planta. (Molano, 2013).

Respecto a las comunidades indígenas, la Universidad del Rosario (s.f.) menciona que:

"son el grupo humano que vive de acuerdo con las formas de relación con el medio natural en el que se asentaron los diferentes grupos aborígenes desde antes de la conquista y la han conservado y dinamizado a lo largo de la historia" (párr.1)

Adicionalmente, es importante mencionar que de acuerdo con los datos suministrados por el Sistema Nacional de Informaciòn Cultural [SINIC], en el Cauca Colombia se encuentran 8 etnias indìgenas: los yanaconas, los ingas, los kokonukos, los totoroes, los paéces, los guambiano, los eperara y los siapidara. Siendo los indìgenas paéces el grupo étnico més numeroso del departamento (SINIC, 2017).

En cuanto al posconflicto, resulta complejo dar una definición exacta; sin embargo, según San Pedro (2006) se puede describir como: “el periodo de tiempo en el cual las hostilidades del pasado se 
han reducido al nivel necesario para que las actividades de reintegración y rehabilitación se puedan iniciar" (citado en Vargas, 2018, p.19). En ese sentido, se requiere principalmente el cese de violencia en el marco de un acuerdo de paz, en el que la sociedad logre superar el daño físico y mental, aprendiendo a olvidar, perdonar y sanar las heridas del pasado, en la práctica de acciones no solo políticas, sino sociales y académicas.

El departamento del Cauca Colombia no ha sido ajeno al flagelo del conflicto armado, especialmente, se observan los efectos generados por la violencia en la población campesina, afrodescendiente y en las comunidades indígenas, quienes siguen solicitando medidas de protección especial, ya que históricamente se han encontrado en medio del fuego cruzado, en constantes abusos y violación a sus derechos humanos, convirtiéndolos en sociedades migrantes.

Tal como lo manifiesta Ávila (2009):

"En el Cauca Colombia han ejercido presencia, además, otros grupos guerrilleros ha habido una presencia histórica de diversos actores armados: las Fuerzas Armadas Revolucionarias de Colombia (Farc), el Ejército de Liberación Nacional (ELN), el Ejército Popular de Liberación (EPL), el Movimiento 19 de Abril (M-19), el Movimiento Quintín Lame, el Movimiento Jaime Bateman Cayón, el Comando Ricardo Franco Frente-Sur, el Partido Revolucionario de los Trabajadores (PRT) y el Comando Pedro León Arboleda" (p.7).

Adicionalmente, según Rangel (2008), desde el año 2005 se vienen presentando acciones de grupos emergentes y rearmados en el departamento del Cauca Colombia, grupos como los Rastrojos y las Águilas Negras se ubican en la zona. Además, este mismo estudio manifiesta que, la Organización Nueva Generación [ONG], otro grupo armado emergente, tiene también incidencia en la zona del Alto Patía en los municipios de Argelia, El Bordo, El Tambo y que, a su vez, los Rastrojos inciden en los municipios costeros de Guapi, Timbiquí y López de Micay, y se relacionan con la presencia del narcotráfico (p.8).

Sumado a lo anterior, el conflicto armado se convirtió en una nube que por décadas escondió otros problemas medulares del departamento del Cauca Colombia y de Colombia, como la concentración de la propiedad rural en manos de hacendados e industriales, las concesiones mineras, la minería ilegal, los conflictos entre indígenas, afros y campesinos por la tierra, y los errores que cometió el Instituto Colombiano de Desarrollo Rural [INCODER] a la hora de comprar y titular predios. 
Con este panorama, a los campesinos, afrodescendientes e indígenas que trabajan habitan y laboran en el campo no solo les preocupa que las tierras más fértiles estén en pocas manos, sino que hasta las zonas hacia donde fueron desplazados, como los páramos y las montañas, han llegado los mineros legales e ilegales a contaminarles los ríos y arrebatarles la poca fertilidad a los suelos.

En consecuencia, todo este contexto desencadenado no solo por el conflicto armado ha provocado que el desarrollo de las actividades productivas de las comunidades indígenas del departamento del Cauca Colombia se haya visto entorpecido, incluso después de firmar el acuerdo de paz con las FARC.

\section{Estado del arte}

Como lo relata De la Peña Begue (1971), en cuanto a la antigüedad del uso de la coca, una idea aproximada es dada por varios autores, al afirmar que en Perú se hallaron bolsas de coca en tumbas a las que, cuando menos se les atribuyen diez siglos. Afirmando tambien, que durante la Colonia el comercio de la coca fue uno de los negocios más lucrativos. Los españoles vieron en su cultivo una de las entradas más ricas del reino y lo aumentaron considerablemente. Pero en aquella época se desconocian sus efectos tóxitos. Este uso de la coca hace referencia al mambeo, a los rituales, usos medicinales y demás constumbres y tradiciones de las comunidades indigenas, de lo cual existe bibliografía abundante; ya que el tema ha sido de mucho interés para las ciencias humanas, como se puede analizar en A.G.I. (1573), Arboleda (1948), Fernandez de Piedrahita (1942) y en Pineda (1986), quienes coinciden en el papel fundamental de la hoja de coca en dichas comunidades.

En cuanto a la temática especifica de usos agroindustriales de la hoja de coca, la bibliografía es escasa, esto tiene mucho que ver con el énfasis que históricamente se ha puesto en la producción, uso y distribución de la cocaína; temática de la cual se encuentra abundante bibliografía, entre ellos: estudios científicos, políticas públicas y memorias de eventos como los escritos por: Saignes (1988), Tovar (1999), Oficina de las Naciones Unidas Contra la Droga y el Delito [UNODC] (2015), entre otros.

Pese a lo anterior, los productos de la hoja de coca diferentes a la cocaína son presentados en congresos, encuentros, eventos de emprendimiento, artículos de periódico, revistas y publicaciones electrónicas; Sin embargo, es un tema muy poco tratado en bibliografía científica. La publicación colombiana más relacionada con la temática y con reconocimiento académico es la realizada por Troyano 
Caicedo-Domínguez., Y.; Hoyos-Garcés., G.; Yépez., R.; Sandoval-Pinedo., D., K.

y Restrepo (2018), en la cual se presentan los horizontes de la industrialización de la hoja de coca con experiencias legales en Perú, Bolivia y algunas experiencias Colombianas.

\section{MÉTODO}

\section{Diseño}

La investigación realizada fue de carácter cualitativo, ya que como lo determina Hernández, Fernández y Baptista (2006), el enfoque cualitativo permite descubrir o afinar preguntas de investigación en el proceso de interpretación, esto se requirió al realizar el estado del arte, el marco legal y estratégico mediante un proceso inductivo; así mismo, en el desarrollo del tercer objetivo, pues se realizó un acercamiento a las comunidades en el que se requirió analizar cada caso, dato por dato, hasta llegar a una perspectiva general. Siguiendo la misma línea teórica, el tipo de investigación fue un estudio exploratorio, ya que se trata de un tema o problema de investigación poco conocido, incluyendo la identificación de posibles variables a estudiar en un futuro.

\section{Participantes}

De las 8 etnias indígenas identificadas por el Sistema Nacional de Informaciòn Cultural [SINIC] (2017) que se encuentran en el Departamento del Cauca Colombia, se eligieron las comunidades Páez y Yanaconas, debido a su arraigo cultural a la hoja de coca.

\section{Instrumentos}

Se utilizó la entrevista a líderes de las diferentes comunidades indígenas del departamento del Cauca Colombia para determinar sus apreciaciones y experiencias respecto a la temática y para la sistematización de experiencias que se están presentando actualmente respecto a los usos agroindustriales de la hoja de coca. La entrevista se realizó personalmente y fue semiestructurada.

\section{Procedimientos}

En el departamento del Cauca Colombia se encontraron experiencias significativas de productos derivados de la hoja de coca, como son; la empresa Coca Nasa, con 25 años de historia; el caso - Lerma, la experiencia en el Servicio Nacional de Aprendizaje [SENA] y otras pequeñas unidades productivas. Estás experiencias se sistematizaron mediante fuentes primarias y fuentes secundarías. 


\section{RESULTADOS}

\section{Coca Nasa}

La empresa Coca Nasa constituye la experiencia más significativa y exitosa en producción y comercialización de productos alternativos agroindustriales de la hoja de coca del departamento del Cauca Colombia y del país, su lucha por permanecer en el mercado y por la defensa de sus productos y costumbres ancestrales lleva 25 años. Coca Nasa es una empresa creada por Fabiola Piñacue y el resguardo indígena de Calderas en Tierradentro Cauca Colombia, nace con el objetivo de cambiar la perspectiva negativa asociada a la hoja de coca y preservar la cultura, tradición étnica y uso de la planta como alimento, medicina, cosmovisión, rito y economía sostenible (Coca Nasa, 2019).

Hasta el momento la empresa ha tenido que sortear grandes obstáculos, entre ellos las regulaciones del Instituto Nacional de Vigilancia de Medicamentos y Alimentos [INVIMA], la Dirección Nacional de Estupefacientes, entidades internacionales e incluso un litigio con la transnacional CocaCola Company por la bebida gaseosa Coca Sek, la empresa reclamaba los derechos de propiedad sobre la palabra Coca, situación que lograron resolver satisfactoriamente puesto que la palabra Coca está arraigada a las culturas indígenas desde hace miles de años (Coca Nasa, 2019).

Actualmente Coca Nasa es la primera y única empresa de alimentos a base de hoja de coca en Colombia que cuenta con permiso para usar esta planta y registro sanitario de sus productos. (Coca Nasa, 2019)

Tabla 1.

Productos elaborados con hoja de coca

\begin{tabular}{lllll}
\hline \multicolumn{1}{c}{ Alimentos } & \multicolumn{1}{c}{ Bebidas } & Coca medicinal & \multicolumn{1}{c}{ Aromáticas } \\
\hline $\begin{array}{l}\text { Harina de Coca } 250 \text { gramos y } \\
120 \text { gramos }\end{array}$ & Aguardiente de Coca & Aceite de Coca & $\begin{array}{l}\text { Aromática de Coca por 20 y por 60 } \\
\text { sobres }\end{array}$ \\
$\begin{array}{l}\text { Galletas de Coca } \\
\text { chocolate }\end{array}$ & con & Vino de Coca & $\begin{array}{l}\text { Gel de Coca } 60 \mathrm{ml} \mathrm{y} \\
120 \mathrm{ml}\end{array}$ & Aromática Coca y Manzanilla \\
& Ron de Coca & Pomada de Coca & Aromática Coca y Menta \\
Hoja de Coca entera & Coca Sek (energética) & Biogotas & Aromática Ecoca (línea económica) \\
\hline
\end{tabular}

Fuente: Coca Nasa (2019). 
Caso SENA

En 2017, el SENA obtuvo el primer permiso en la historia de Colombia para realizar investigación científica con la hoja de coca, otorgado por el Fondo Nacional de Estupefacientes, permiso que da lugar a la compra local de hoja de coca por el SENA a comunidades autorizadas, que son las que la cultivan para usos tradicionales y al uso exclusivo de la planta para fines de investigación (Troyano y Restrepo, 2018).

En el marco del programa del sistema de Investigación, Desarrollo Tecnológico e Innovación [SENNOVA] del SENA, las investigadoras Dora Lucila Troyano y María del Socorro Anaya desarrollaron un proyecto de investigación cuyo resultado fue la producción tecnificada de abonos orgánicos sólidos y líquidos, a partir de la hoja de coca, para fertilización de cultivos transitorios. Las investigadoras desarrollaron un procedimiento para elaborar estos abonos, el cual fue sistematizado en una cartilla (Anaya y Troyano, 2017). Esta cartilla, es compartida como apoyo a la capacitación de los campesinos de los diferentes municipios del departamento del Cauca Colombia, con el fin de lograr que el material vegetal disponible en las zonas cocaleras sea aprovechado por las comunidades como materia prima para la elaboración de abonos. Lo anterior, teniendo en cuenta que la fórmula es más fácil y económica que las opciones de abonos químicos que se encuentran en el mercado y que su implementación garantizaría lograr una producción orgánica de los cultivos.

\section{La Ruta de la Coca en Lerma}

Lerma está ubicado al sur del departamento del Cauca Colombia y pertenece al municipio de Bolívar, se trata de un corregimiento muy golpeado por las nefastas consecuencias de la bonanza cocalera, de lo que dan fe algunos líderes entrevistados para esta investigación. En el año de 1983 el corregimiento se encontraba con los más altos niveles de homicidio y muertes violentas, correspondiente al $25 \%$ de una población que no superaba los 500 habitantes. Gracias a procesos comunitarios fuertes y continuos se generó un cambio y se logró la declaración por la asamblea departamental del municipio como Territorio de Convivencia y Paz (Corregimiento de Lerma, 2019). Entre los líderes que lograron estos procesos se encuentra Erney Ruiz, líder de la Escuela Agroambiental Arraigo y quien lidera los procesos de productos alternativos con hoja de coca, como la ruta de la coca, en alianza con el SENA, el apoyo de la investigadora Dora Troyano y la Open Society Foundations. 
Actualmente, este proyecto cuenta con un grupo de alrededor de 20 campesinos, quienes venden la coca producida de manera orgánica a la Escuela Agroambiental Arraigo, para que sea transformada en los siguientes productos:

○ Galletas

○ Harina

○ Caucharina (pasaboca dulce en polvo)

○ Pomada

○ Tortas

○ Hojas secas

Además, se creó una unidad productiva para implementar "la ruta de la coca", se trata de una opción turística que se brinda a los visitantes en la cual se realizan caminatas ecológicas por charcos, cerros y un recorrido por la historia de la hoja de la coca, sus usos medicinales, nutricionales y sus características ancestrales y espirituales (El nuevo liberal, 2019).

Por su parte, Erney Ruiz considera que la producción y comercialización de productos de hoja de coca trae múltiples ventajas, como ser amigable con el medio ambiente y generar una economía estable que permite unidad entre la ruralidad y lo urbano. Esto les ha permitido la resistencia en el territorio y autonomía “desde hace 15 años dejamos de ser jornaleros de otros, ahora no dependemos y damos trabajo, participación social con recursos propios".

Para Erney Ruiz, el camino correcto es seguir con el proyecto hasta convertirla en una empresa productora y comercializadora de productos agroindustriales basados en la hoja de coca, pero en la legalidad. Cabe resaltar, que este proyecto no cuenta hasta el momento con los permisos y el reconocimiento legal para la producción y comercialización de estos productos.

En el departamento existen otras experiencias que generan productos de hoja de coca, pero realmente no son muy significativas. En la vereda Segovia de Inzá, se entrevistó a la señora Cilia Narváez quien, en compañía de su esposo, produce vino artesanal de coca usado principalmente para eventos especiales en la comunidad. Para la señora Cilia, esta pequeña unidad productiva le ha permitido generar ingresos para el estudio de sus hijos; sin embargo, ella manifestó que le gustaría tener una planta de producción y el registro del cabildo indígena para poder comercializar su producto libremente. 
Durante la investigación se realizaron entrevistas a líderes en comunidades indígenas en el municipio de Inza, Corinto y Toribio con el fin de identificar otras experiencias significativas de producción alternativa basada en hoja de coca, pero, sobre todo, para conocer el interés de estas comunidades en crear unidades productivas. El resultado encontrado fue, que el interés de las comunidades por este tipo de productos es muy bajo, debido a que siguen manteniendo un arraigo cultural a la coca enfocada en rituales y procesos medicinales, pero no como una opción productiva. Una de las razones encontradas, son los problemas a los que se tuvieron que enfrentar durante muchos años las comunidades, por mantener este tipo de cultivos. Otra razón importante es la adherencia a programas de sustitución.

\section{Contexto Jurídico}

Con el análisis propio de la legislación colombiana, el cultivo del arbusto de coca es prohibido, sin embargo, existen dos formas de trabajar la coca de una manera legal:

1. En la declaración segunda de la Ley 67 (1993) se dispone que:

“el tratamiento que la Convención da al cultivo de la hoja de coca como infracción penal debe armonizarse con una política de desarrollo alternativo, tomando en cuenta los derechos de las comunidades indígenas involucradas y la protección del medio ambiente..."

En la anterior declaración, se encuentra una notable desigualdad en cuanto al cultivo de coca, pues se especifica que solo las comunidades indígenas pueden realizar dicha actividad, dejando por fuera a personas mestizas que en muchos casos también realizan este tipo de cultivos con el fin de generar opciones lícitas que contribuyen al crecimiento y enriquecimiento de la cultura, el empleo y la aplicación de nuevas actividades en la sociedad.

2. Por otra parte, la Convención Única Sobre Estupefacientes (1961), señala en su artículo 27 que:

"Es autorizado el uso de hojas de coca para la preparación de un agente saporífero (que da sabor) que no contenga ningún alcaloide y, en la medida necesaria para dicho uso, autorizar la producción, importación, exportación, el comercio y la posesión de dichas hojas" (p.33) 
Adicionalmente, de acuerdo con Naciones Unidas (s.f.):

"Las Partes suministrarán por separado previsiones e información estadística respecto de las hojas de coca para la preparación del agente saporífero, excepto en la medida en que las mismas hojas de coca se utilicen para la extracción de alcaloides y del agente saporífero y así se explique en la información estadística y en las previsiones" (p.35)

Por otra parte, en atención a lo dispuesto por el artículo 3ro de la Convención de las Naciones Unidas contra el tráfico ilícito de estupefacientes y sustancias sicotrópicas (1988), se hace mención al catálogo de conductas que pueden ser tipificadas como delitos penales, entre ellas "el cultivo del arbusto de coca $[. .$.$] con objeto de producir estupefacientes..." (p.3). En lo manifestado anteriormente, se plasma$ de forma implícita que mientras se cultive el arbusto de la hoja de coca con fines agroindustriales no se está incurriendo en ningún delito por no producir estupefacientes con ésta.

Por los puntos expuestos anteriormente, es evidente que existe la normatividad que regula la siembra y cultivo de la coca para fines lícitos; de la misma forma, se puede observar que la divulgación de dichos preceptos no es la adecuada, pues aún persiste desinformación y temor en las comunidades respecto a la licitud con la que se puede trabajar esta planta.

Adicionalmente, es alarmante que las autoridades desatiendan un tema tan importante como lo es el cultivo de la hoja de coca, teniendo en cuenta que hace parte de la flora nativa de Colombia, de las tradiciones ancestrales de los pueblos indígenas y campesinos del país, y finalmente, por sus múltiples utilidades alternativas, desde lo artesanal, económico e incluso gastronómico.

En una de las declaraciones que el Gobierno presentó, mediante la Corte Constitucional Colombiana y el Control Previo de los Tratados Internacionales 1192 - 2012, al Congreso de la República, plasmó que:

"Colombia entiende que el tratamiento que la Convención da al cultivo de la hoja de coca como infracción penal debe armonizarse con una política de desarrollo alternativo, tomando en cuenta los derechos de las comunidades indígenas involucradas y la protección del medio ambiente. En el mismo sentido Colombia entiende que el trato discriminatorio, inequitativo y restrictivo que se les da en los mercados internacionales a sus productos agrícolas de exportación, 
Caicedo-Domínguez., Y.; Hoyos-Garcés., G.; Yépez., R.; Sandoval-Pinedo., D., K.

en nada contribuye al control de los cultivos ilícitos pues, por el contrario, es causa del deterioro social y ecológico en las zonas afectadas.” (Citado en Ceron y Arevalo, 2014, p.54)

En ese orden de ideas, la solución al conflicto es la creación de sociedades mercantiles apegadas a las leyes que las regulan, para que una vez formadas (legalmente) puedan trabajar como empresa con los indígenas (como agentes productores de materia prima) y de esta forma, los beneficios serían tanto para la sociedad en general como para las comunidades. Lo anterior, debido a que se pone en evidencia la necesidad del trabajo en equipo de cada uno de los actores.

Por lo anterior, Colombia, como Estado social de derecho y garante de los mismos, en su Constitución Política (1991) , manifiesta que: "Se garantiza el derecho de libre asociación para el desarrollo de las distintas actividades que las personas realizan en sociedad" (art. 38)

Adicionalmente, entre los antecedentes que se pueden encontrar en el marco jurídico colombiano, respecto a la hoja de coca, se presenta en la siguiente línea del tiempo el resultado del trabajo de Troyano (2018), entrevistada para esta investigación

Figura/Ilustración 1

\section{Línea de tiempo}

\begin{tabular}{|c|c|c|c|}
\hline $\begin{array}{l}\text { Libre siembra de arbusto de } \\
\text { coca, con ciertas restricciones. }\end{array}$ & $\begin{array}{l}\text { Represión al cultivo y } \\
\text { conservación de coca. }\end{array}$ & $\begin{array}{l}\text { Establece un espacio de } \\
\text { legalidad a los cultivos de coca } \\
\text { en comunidades indígenas. }\end{array}$ & $\begin{array}{l}\text { Consumo de la hoja de coca } \\
\text { como parte del patrimonio } \\
\text { cultural inmaterial de los }\end{array}$ \\
\hline Ley 11 de 1920 & Ley 45 de 1946 & $\begin{array}{l}\text { Convención de la ONU } \\
\text { de Viena } 1988\end{array}$ & Ley 397 de 1997 \\
\hline 1936 & 1946 & 1991 & 1997 \\
\hline Código Penal de 1936 & Decreto 0896 de 1947 & Convenio 169 de la OIT & Código Penal de 2000 \\
\hline $\begin{array}{l}\text { No penalizaba el cultivo de } \\
\text { coca, ni el consumo de } \\
\text { alcaloides }\end{array}$ & $\begin{array}{l}\text { Prohibió la utilización de la hoja } \\
\text { de coca como moneda. }\end{array}$ & $\begin{array}{l}\text { Establece protección a la cultura } \\
\text { (costumbres y tradiciones) de } \\
\text { los indígenas. }\end{array}$ & $\begin{array}{l}\text { Establece sanciones para } \\
\text { plantaciones del arbusto de coca } \\
\text { (art. 375) }\end{array}$ \\
\hline
\end{tabular}


En la zona de Tierra dentro, se otorga permiso para utilizar la hoja de coca para aromáticas.
Se informa: "Productos base de la coca, elaborados y comercializados por indígenas, no se podrán comercializar más en territorio nacional."

Resolución 001 de la Asociación de Cabildos Juan Tama. Inzá, Cauca Colombia.
Circular INVIMA V.C.M-601-0294-07

Circular INVIMA V.C.M-601-0294-07
Resolución 1478 del Ministerio de Protección Social

En la lista de sustancias objeto de monopolio del Estado, se incluye la hoja de coca y derivados.

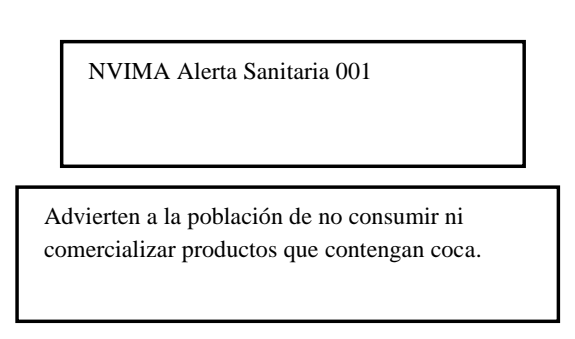

Fuente: Troyano (2018).

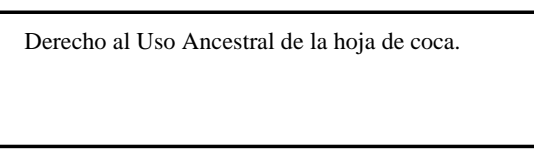

Corte Constitucional C-882

\section{5}

Consejo de Estado. Sala de lo

Contencioso Administrativo

Se decreta acción de nulidad frente a la alerta sanitaria 001 de 2010.

Además, un hecho importante a nivel internacional es el ocurrido hacia el año 2011, cuando Bolivia se retiró de la Convención de Viena, de la que fuera parte por más de cincuenta años y en el mismo año solicitó su readmisión, pero esta vez con la condición de que el acullicu (bola de hojas de coca que se mastica para extraer el jugo de las mismas) estuviera despenalizado, con dicha readmisión, señaló el Jefe de Estado Plurinacional, que se tiene como ventaja la "permisibilidad de cultivar la hoja de coca para usos tradicionales".

$\mathrm{Al}$ respecto, Contreras-Baspineiro (2013) señaló que: “Casi 50 años, la coca estuvo penalizada por decisiones políticas de los países desarrollados que son los que controlan las decisiones en el mundo, hoy Bolivia, tuvo la capacidad de escribir una digna página de dignidad, soberanía y justicia" (p.16). Lo que propone es un tema muy interesante, con lo cual se llegó a la interrogante de ¿quiénes son las personas que hacen mal uso de la coca y a quiénes no les conviene que se cultive?

Sin embargo, la Oficina de Naciones Unidas Contra la Droga y el Delito (2015), señala que actualmente Bolivia produce 27.200 hectáreas de coca, siendo que la Ley 1008 (2006), reconoce 12 mil hectáreas de coca como lícitas, por lo que se pone en cuestión si en verdad el doble de lo permitido es necesario para cubrir los usos meramente tradicionales o medicinales. Y aunado a esto, Bolivia aún no cuenta con un estudio que determine la cantidad de cultivos de coca para usos tradicionales y alternativos, como si lo ha hecho Colombia. 


\section{Discusión}

El departamento del Cauca Colombia es un departamento agrario, llamado "la despensa agraria de Colombia", sin embargo, no es evidente la rentabilidad de esta actividad económica para las comunidades del departamento. Tanto así, que se pueden encontrar comunidades que viven la violencia exacerbada en el último año, campesinos pobres, y permanente descontento de las comunidades indígenas, al punto de cerrar las vías de acceso al departamento como última medida para solicitar al gobierno el cumplimiento de derechos ganados y de acuerdos realizados previamente. Otro aspecto evidente durante la investigación es la falta de agroindustria y de empresas productivas, pues es claro que se requiere la transformación de la riqueza agraria en productos que generen verdadero valor agregado, desarrollo empresarial y las tan anheladas fuentes de empleo. Además, se pone en evidencia el latente el abandono del estado, palpable en las vías de acceso y la baja rentabilidad en el cultivo de productos agrarios que contribuyen a la alta producción de la hoja de coca para fines ilícitos.

El estudio motiva a fomentar y apoyar una agroindustria limpia, para que de una materia prima tan rica como la hoja de coca sea posible darle paso a la producción y comercialización de productos agroindustriales alternativos, que la legislación actual no permite y que tampoco están contemplados dentro de lo establecido en las políticas de postconflicto.

Es lamentable que solamente las comunidades indígenas por su legislación especial y por el caso de Coca Nasa pueden pensar en la posibilidad de reivindicar a la hoja de coca a través de la gran cantidad de productos alimenticios y medicinales que se podrían desarrollar y es aún más preocupante que, según los resultados del estudio, se observa que las comunidades no están interesadas en producirlos, pues para ellos implica luchar contra los dueños del negocio de la cocaína, contra el paradigma social que aún no entiende la diferencia entre hoja de coca y la cocaína, y además contra el estado actual que prefiere la erradicación con glifosato por ser el camino más fácil, aunque sea también el más dañino para la comunidad.

Las comunidades indígenas no están dispuestas a poner más muertos y tampoco a seguir sufriendo la discriminación y abandono estatal, pero lo que si es cierto es que no se van a desarraigar de la base de sus ritos, de su espiritualidad, del legado de sus ancestros y de los beneficios medicinales y nutricionales de esta planta, seguirán teniéndola solo para ellos, sin poderla compartir en el resto del territorio nacional y menos internacionalmente. 


\section{CONCLUSIONES}

El trabajo investigo permite encontrar el arraigo histórico de la coca en las comunidades indígenas, su uso alimenticio, medicinal y cultural, así como el empleo de los principios activos de la coca y los productos derivados, con ello se identifican los usos agroindustriales de la hoja de Coca.

Los casos expuestos demuestran la viabilidad de la agroindustria proveniente de la hoja de coca, teniendo en cuenta las características del cultivo, las condiciones para el proceso de producción, la diversidad en los productos y los estudios que avalan las riquezas de sus componentes.

Si bien existen leyes que regulan el cultivo ilícito, tanto el gobierno nacional como diferentes países apoyan tanto las políticas como los programas de postconflicto, estos deberían ser tendientes a incentivar el uso medicinal y de productos de la coca, por parte de las comunidades que han sido afectadas por el conflicto armado en Colombia. Pero contrario a lo anterior, el principal impedimento para generar esta opción de desarrollo económico y productivo de la región es el contexto político y legal, que no solamente juega con intereses del país sino con una gran presión internacional, factores que impiden contemplar esta alternativa en las políticas y programas del postconflicto.

El trabajo investigativo que se ha realizado sobre la hoja de coca es altamente relevante, se encuentran estudios fundamentados y de gran rigurosidad metodológica que han logrado un proceso de reivindicación con esta planta tan arraigada a la cultura y costumbres de las comunidades indígenas de Colombia, Perú y Bolivia. Además, se ha logrado desviar la mirada del pequeño productor a la gran organización ilegal de la cocaína que es realmente donde está la causa del flagelo de violencia y degradación social de la que injustamente se culpó por mucho tiempo a "la mata que mata".

Con este cambio de paradigma se abren múltiples posibilidades, que pueden llevar al país a convertir lo que por años fue causa de desgracia, en una fuente de desarrollo rural, económico y productivo, pero sobre todo ayudará a favorecer a las comunidades indígenas que se encuentran actualmente en condiciones de vulnerabilidad.

Casos exitosos como la empresa Coca Nasa en producción y comercialización de productos alternativos agroindustriales de la hoja de coca, permiten vislumbrar que con el apoyo en el plan de negocios y asesoría técnica y administrativa se puede mejorar tanto la comercialización como la expansión de la empresa. (recomendación) 
Caicedo-Domínguez., Y.; Hoyos-Garcés., G.; Yépez., R.; Sandoval-Pinedo., D., K.

El estudio desarrollado cumple con los objetivos planteados y permite a futuro apoyar en la parte comercial y administrativa en el surgimiento de empresas agroindustriales con productos derivados de la coca, así como la conservación de la cultura indígena de las regiones del departamento del Cauca Colombia.

\section{REFERENCIAS}

Anaya, M., Troyano, D. (s.f.). Guía producción tecnificada de abonos orgánicos sólidos y líquidos a partir de la hoja de coca para fertilización de cultivos transitorios. Servicio Nacional de Aprendizaje SENA [Archivo PDF]. https://es.scribd.com/document/428670149/Troyano-yAnaya-Abonos-de-Coca-SENA-Cauca Colombia-Noviembre2017

Arboleda, J. (1948). El indio en la Colonia. Ministerio de Educación Nacional.

Avendaño Avendaño, M., Galvis Pérez, A. E. y Velasco Hurtado, D. E. (2019). Retos y desafíos de las actividades productivas en las comunidades indígenas del departamento del Cauca Colombia en el marco del posconflicto. Revista Estrategia Organizacional, 8 (1). doi: https://doi.org/10.22490/25392786.3176

Ávila, A. (2009) Conflicto Armado en Cauca Colombia: Reconfiguración del poder regional de los actores armados. Corporación Nuevo Arco Iris.

Cerón, K., Arévalo, W.( 2014). Centro de Estudios Publicos e Internacionales. De la Constitución de 1991 a la realidad. Debates políticos, jurídicos, territoriales e internacionales. Universidad del Rosario

COCA-NASA (s.f.). Productos Cosa Nasa. https://cocanasa.org/blogs/5d111f6b568ba3538d91c347

Coca (planta). (24 noviembre 2020). En EcuRed. Recuperado el 17 de diciembre de 2020. https://www.ecured.cu/index.php?title=Coca_(planta)\&oldid=3810542.

Constitución Política de Colombia [Const.] Artículo 38. 7 de julio de 1991. Colombia.

Combita, L., Delgadillo, L. y Torres S. El posconflicto en Colombia: una mirada hacia los diferentes procesos de paz. [Tesis en Especialización en Gerencia Social, Corporación Universitaria Minuto de Dios. Facultad Ciencias Empresariales]. https://repository.uniminuto.edu/bitstream/handle/10656/2224/TEGS_CombitaLuzMery_2013. pdf? sequence $=1 \&$ isAllowed $=\mathrm{y}$

Contreras, A. (13 de enero de 2013). La hoja de coca triunfó en el mundo. [entrada de blog]. https://www.alainet.org/es/active/60930 
Corregimiento de Lerma. (14 de abril de 2018). Corregimiento de Lerma [entrada de blog]. http://corregimientodelerma.blogspot.com/

Decreto 896 de 1947 [Ministerio de la Protección Social]. En desarrollo del parágrafo $1^{\circ}$ del artículo $5^{\circ}$ de la Ley $6^{\mathrm{a}}$ de 1945 , del ordinal $1^{\circ}$ del artículo 27 del Decreto 2127 de 1945 y del artículo $1^{\circ}$ de la Ley 45 de 1946. 11 de marzo de 1947. DIARIO OFICIAL. 26387

De La Peña, R. (1971). Antropología Americana. El uso de la coca en América Latina, según la legislación colonial y republicana. Madrid: Universidad de Madrid, 1971 [citado 05 de junio de 2019]. https://dialnet.unirioja.es/servlet/articulo?codigo=901657.

Fernandez, L. (1942). Historia General del Nuevo Reino de Granada. Biblioteca Popular de Cultura Colombiana.

Hernández, R., Fernández, C. y Baptista, P. (2006). Metodología de la investigación. McGraw-Hill. Instituto Nacional de Vigilancia de Medicamentos y Alimentos [INVIMA] (2019). Alerta Sanitaria 001. Dirección de Dispositivos Médicos y Otras Tecnologías. https://app.invima.gov.co/alertas/ckfinder/userfiles/files/ALERTAS\%20SANITARIAS/Disposit ivos_Medicos/2019/Enero/Alerta\%20No\%23001-2019\%20-

\%20Guante\%20de\%20L\%C3\%A1tex\%20Quir\%C3\%BArgico\%20Est\%C3\%A9ril\%20Nipro\% 20.pdf

La ruta de la coca, proyecto turístico (21 de noviembre de 2017). El Nuevo Liberal. https://elnuevoliberal.com/la-ruta-de-la-coca-proyecto-turistico/

Ley 1008 de 2006. Por la cual se fijan algunas competencias y procedimientos para la aplicación de convenios internacionales en materia de niñez y de familia. 23 de enero, 2006. Diario oficial. $\mathrm{N}^{\circ} 46.160$.

Ley 11 de 1920. Sobre importación y venta de drogas que formen hábito pernicioso. 15 de septiembre de 1920. DIARIO OFICIAL. 17.322.

Ley 397 de 1997. Por la cual se desarrollan los Artículos 70, 71 y 72 y demás Artículos concordantes de la Constitución Política y se dictan normas sobre patrimonio cultural, fomentos y estímulos a la cultura, se crea el Ministerio de la Cultura y se trasladan algunas dependencias. 7 de agosto de 1997. DIARIO OFICIAL. 43102.

Ley 45 de1946. Por la cual se subrogan algunas disposiciones de los Códigos Penal y de Procedimiento Penal y se adicional otras de la Ley 167 de 1941. 18 de diciembre de 1946. DIARIO OFICIAL. 26311.

Ley 599 de 2000. Por la cual se expide el Código Penal. 24 de julio de 2000. DIARIO OFICIAL. 44.097. 
Caicedo-Domínguez., Y.; Hoyos-Garcés., G.; Yépez., R.; Sandoval-Pinedo., D., K.

Ley 67 de 1993. Por medio de la cual se aprueba la "Convención de las Naciones Unidas contra el tráfico ilícito de estupefacientes y sustancias sicotrópicas. 23 de agosto de 1993. DIARIO OFICIAL. 41.003 .

Ley 95 de 1936. Sobre el Código Penal. 24 de abril de 1936. DIARIO OFICIAL. 23316.

Molano, A. (25 de agosto de 2013). Otras caras de la coca. El Espectador. https://www.elespectador.com/noticias/politica/otras-caras-de-coca-articulo-442394

Naciones Unidas (1988). Convención de las Naciones Unidas contra el tráfico ilícito de estupefacientes y sustancias sicotrópicas [Archivo PDF]. https://www.unodc.org/pdf/convention_1988_es.pdf

Naciones Unidas (s.f.). Convención Única de 1961 Sobre Estupefacientes [Archivo PDF].. https://www.incb.org/documents/Narcotic-Drugs/1961-Convention/convention_1961_es.pdf

Oficina de las Naciones Unidas Contra la Droga y el Delito [UNODC] (2015). Monitoreo de Cultivos de Coca 2014 [Archivo PDF]. $\underline{\text { https://www.unodc.org/documents/crop- }}$ monitoring/Colombia/Colombia_Monitoreo_de_Cultivos_de_Coca_2014_web.pdf

Organización Internacional del Trabajo [OIT] (1989). Convenio 169. Sobre pueblos indígenas y tribales en países independientes. https://www.refworld.org.es/docid/50ab8efa2.html

Pineda, R.(1986). Etnografía del Mambeadero. Espacio de la coca. Rev. Texto y Contexto, (9), 113-127.

Rangel, A. (2008). Perspectivas de paz y seguridad. Rev. Criminalidad, 50 http://www.scielo.org.co/scielo.php?script=sci_arttext\&pid=S1794-31082008000100015

Resolución 001 de 2002 [Asociación de Cabildos Juan Tama]. Por medio de la cual se impone una multa en la ejecución del contrato IDCT 17-0099-00-0. 11 de diciembre de 2002.

Resolución 1478 de 2006 [Ministerio de la Protección Social]. Por la cual se expiden normas para el control, seguimiento y vigilancia de la importación, exportación, procesamiento, síntesis, fabricación, distribución, dispensación, compra, venta, destrucción y uso de sustancias sometidas a fiscalización, medicamentos o cualquier otro producto que las contengan y sobre aquellas que son monopolio del Estado. 10 de mayo de 2006. DIARIO OFICIAL. 46292

Rojas, F. (2002). La economía de la coca. [Trabajo de grado, Instituto de Investigaciones Socioeconómicas, Universidad Católica Boliviana]

Saignes, C., Potosi C. (1988). El consumo popular de estimulantes en el siglo XVII. Rev de Indias. 48, 182-183. http://www.bibvirtual.ucb.edu.bo:8000/etnias/digital/106001195.pdf

Sentencia C-882 [Corte Constitucional, Sala Plena]. Reforma constitucional introducida al artículo 49 de la Constitución política. 23 de noviembre de 2011. 
Sistema Nacional de Información Cultural [SINIC]. (2017). Colombia Cultural. http://www.sinic.gov.co/SINIC/ColombiaCultural/ColCulturalBusca.aspx?AREID=3\&SECID= $\underline{8 \& \mathrm{IdDep}=19 \& \text { COLTEM }=216}$

Tovar, H. (1999). Colombia: Droga, economía, guerra y paz. Planeta Colombiana Editorial S.A.

Troyano, D. y Restrepo, D. (2018). La industrialización de la Hoja de Coca, un camino de innovación, desarrollo y paz en Colombia. Fundaciones Sociedad Abierta.

Universidad del Rosario. (s.f.). Pueblos indígenas. [Entrada de Blog]. https://www.urosario.edu.co/jurisprudencia/catedra-viva-intercultural/ur/Comunidades-Etnicasde-Colombia/Pueblos-indigenas/

Vargas, M. (2018). La ley de restitución de tierras y su efectividad en el municipio de Puerto Asís, departamento del Putumayo. [Tesis de Maestría, Universidad Santiago de Cali. Facultad de $\begin{array}{llll}\text { Ciencias Económicas } & \text { y }\end{array}$ https://repository.usc.edu.co/bitstream/20.500.12421/230/1/LA\%20LEY\%20DE\%20RESTITU CI\%C3\%93N.pdf 